\title{
Dissecting tumor metabolic heterogeneity: Telomerase and large cell size metabolically define a sub-population of stem- like, mitochondrial-rich, cancer cells
}

\author{
Rebecca Lamb ${ }^{1,2}$, Bela Ozsvari ${ }^{1,2}$, Gloria Bonuccelli, ${ }^{1,2}$, Duncan L. Smith ${ }^{3}$, Richard \\ G. Pestell ${ }^{4}$, Ubaldo E. Martinez-Outschoorn ${ }^{4}$, Robert B. Clarke ${ }^{1}$, Federica Sotgia ${ }^{1,2}$ \\ and Michael P. Lisanti ${ }^{1,2}$ \\ ${ }^{1}$ The Breast Cancer Now Research Unit, Institute of Cancer Sciences, University of Manchester, Manchester, UK \\ ${ }^{2}$ The Manchester Centre for Cellular Metabolism (MCCM), Institute of Cancer Sciences, University of Manchester, Manchester, \\ UK \\ ${ }^{3}$ The Cancer Research UK Manchester Institute, University of Manchester, Manchester, UK \\ ${ }^{4}$ The Sidney Kimmel Cancer Center, Philadelphia, PA, USA \\ Correspondence to: Rebecca Lamb, email: rebecca.lamb@ics.manchester.ac.uk
}

Federica Sotgia, email: federica.sotgia@ics.manchester.ac.uk

Michael P. Lisanti, email: michael.lisanti@ics.manchester.ac.uk

Keywords: hTERT, telomerase, cell size, mitochondrial biogenesis, cancer stem cells, proteomic analysis, tumor metabolism Abbreviations: CSCs, cancer stem-like cells, TICs, tumor-initiating cells

Received: June 16, $2015 \quad$ Accepted: July 13, $2015 \quad$ Published: August 27, 2015

This is an open-access article distributed under the terms of the Creative Commons Attribution License, which permits unrestricted use, distribution, and reproduction in any medium, provided the original author and source are credited.

\section{ABSTRACT}

Tumor cell metabolic heterogeneity is thought to contribute to tumor recurrence, distant metastasis and chemo-resistance in cancer patients, driving poor clinical outcome. To better understand tumor metabolic heterogeneity, here we used the MCF7 breast cancer line as a model system to metabolically fractionate a cancer cell population. First, MCF7 cells were stably transfected with an hTERT-promoter construct driving GFP expression, as a surrogate marker of telomerase transcriptional activity. To enrich for immortal stem-like cancer cells, MCF7 cells expressing the highest levels of GFP (top 5\%) were then isolated by FACS analysis. Notably, hTERTGFP(+) MCF7 cells were significantly more efficient at forming mammospheres (i.e., stem cell activity) and showed increased mitochondrial mass and mitochondrial functional activity, all relative to hTERT-GFP(-) cells. Unbiased proteomics analysis of hTERT-GFP(+) MCF7 cells directly demonstrated the over-expression of 33 key mitochondrial proteins, 17 glycolytic enzymes, 34 ribosome-related proteins and 17 EMT markers, consistent with an anabolic cancer stem-like phenotype. Interestingly, MT-CO2 (cytochrome c oxidase subunit 2; Complex IV) expression was increased by $\mathbf{2 0}$-fold. As MT-CO2 is encoded by mt-DNA, this finding is indicative of increased mitochondrial biogenesis in hTERT-GFP(+) MCF7 cells. Importantly, most of these candidate biomarkers were transcriptionally over-expressed in human breast cancer epithelial cells in vivo. Similar results were obtained using cell size (forward/side scatter) to fractionate MCF7 cells. Larger stem-like cells also showed increased hTERTGFP levels, as well as increased mitochondrial mass and function. Thus, this simple and rapid approach for the enrichment of immortal anabolic stem-like cancer cells will allow us and others to develop new prognostic biomarkers and novel anti-cancer therapies, by specifically and selectively targeting this metabolic sub-population of aggressive cancer cells. Based on our proteomics and functional analysis, FDAapproved inhibitors of protein synthesis and/or mitochondrial biogenesis, may represent novel treatment options for targeting these anabolic stem-like cancer cells. 


\section{INTRODUCTION}

Telomerase plays a central role both in the biology of normal aging, as well as in the development of human cancers $[1,2]$. However, its exact role still remains poorly understood. Telomerase is a ribo-nucleoprotein, which contains both telomerase RNA (TERC) and telomerase reverse transcriptase (TERT) subunits [3]. Importantly, the over-expression of telomerase in both normal stem cells and cancer cells is sufficient to confer cell immortalization, such that these cells can divide beyond 50-70 divisions and bypass senescence [4-6].

Recently, Clarke and colleagues have taken advantage of the properties of human telomerase (hTERT), to enrich for a population of osteosarcoma cells with stemlike properties [7]. For this purpose, they fused a $1.5-\mathrm{kB}$ fragment of the hTERT promoter to GFP, in order to select a sub-population of osteosarcoma cells with hightelomerase transcriptional activity by FACS analysis. More specifically, they demonstrated that these hTERThigh-activity osteosarcoma cells showed increased stemlike activity, anchorage-independent growth, invasiveness and metastatic activity, as well as chemo-therapy resistance [7]. As such, this hTERT-based approach for the purification of cancer stem-like cells (CSCs) has already been functionally validated.

Here, we have adapted this approach to the study of breast cancer stem-like cells, with a focus on proteomics analysis, biomarker discovery and cell metabolism. Importantly, we demonstrate that hTERT-high-activity breast cancer cells form mammospheres with higher efficiency, and show a proteomics profile consistent with an anabolic cancer stem cell phenotype. In support of this notion, we also show that hTERT-high-activity breast cancer cells have increased mitochondrial mass and activity, consistent with an increase in mitochondrial biogenesis.

\section{RESULTS}

\section{Experimental approach: fluorescent enrichment of breast cancer stem cell activity}

To enrich for a population of immortal CSCs, we exploited a sensitive eGFP reporter system for the fluorescent detection of high telomerase transcriptional activity. Briefly, MCF7 cells were transduced with a lentiviral vector driving eGFP protein expression, under the control of a $1.5-\mathrm{kB}$ fragment of the hTERT promoter. This DNA construct also contains a puromycin-resistance cassette for antibiotic-resistance selection. Schematic diagrams illustrating this overall experimental strategy and the construction of the hTERT-promoter-vector are shown in Figures 1 and 2.
After selection with puromycin, MCF7-hTERTeGFP cells were subjected to FACS analysis to visualize the broad distribution of eGFP expression, which serves as a surrogate marker of telomerase activity. Importantly, fewer than 1 in a 100 cells in MCF7-hTERT-eGFP cell monolayers visually showed high GFP fluorescence. In striking contrast, there was a dramatic enrichment of GFP $(+)$ cells in MCF7 cell mammospheres $(>50 \mu \mathrm{m})$, each containing usually 1-to-2 GFP-high cells (Figure 2).

To validate the enrichment of mammosphere forming cells, MCF7-hTERT-eGFP cells were fractionated into GFP-high (top 5\%) and GFP-low (negative) groups. Then, five thousands cells from each group were seeded per well in 6-well low-attachment plates. These two groups were compared to the total unfractionated cell population.

Remarkably, Figure 3 shows that GFP-high cells form mammospheres with an efficiency nearly 2.5-fold greater than GFP-negative cells and the total unfractionated cell population. Importantly, in this context, mammosphere formation was sensitive to the administration of a well-characterized telomerase inhibitor, namely MST-312, as expected (Figure 3).

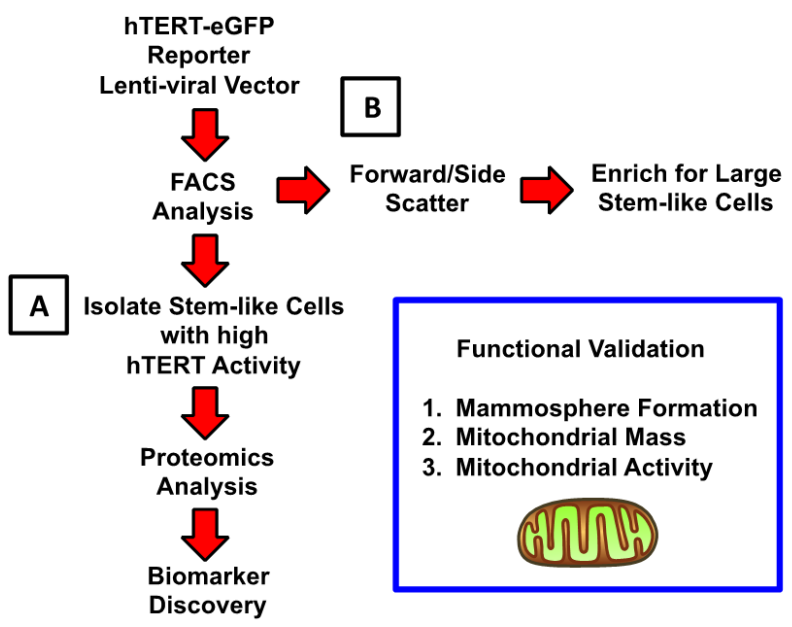

Figure 1: Summary of the overall experimental approach, using FACS analysis, to enrich for breast cancer stem-like cells: Focus on telomerase and cell size. A. Telomerase Activity: To enrich for a population of immortal CSCs, we exploited a sensitive reporter system for the fluorescent detection of high telomerase transcriptional activity (hTERT-eGFP). We used this simplified approach to drive biomarker discovery, via unbiased label-free proteomics analysis. B. Cell Size (Forward Scatter/Side Scatter): Alternatively, we fractionated MCF7 cells based on forward/side scatter into larger and smaller cell populations. Previous studies using mouse mammary epithelial cells have demonstrated that stem-like cells can be enriched solely based on cell size [11]. For example, large stem-like cells with diameters $>10 \mu \mathrm{m}$, defined by higher forward scatter during FACS analysis, showed a $>4$ fold increased ability to undergo mammosphere formation. 
Table 1. Key Molecules Up-regulated in hTERT-GFP(+) MCF7 Cells Isolated by FACS: Mitochondria and Glycolysis.

\begin{tabular}{|c|c|c|c|}
\hline Symbol & Description & $\begin{array}{l}\text { Fold-Upregulation } \\
(\mathrm{GPF}(+) / G \mathrm{FP}(-))\end{array}$ & ANOVA \\
\hline \multicolumn{4}{|c|}{ Mitochondrial-Related Proteins (33) } \\
\hline $\mathrm{MT}-\mathrm{CO} 2$ & Cytochrome c oxidase subunit 2 , mt-DNA encoded & 20.48 & 7.27E-07 \\
\hline GRPEL1 & GrpE protein homolog 1, mitochondrial & 11.90 & 0.006 \\
\hline ECHS1 & Enoyl-CoA hydratase, mitochondrial & 8.64 & $9.13 \mathrm{E}-06$ \\
\hline PARK7 & Protein DJ-1 & 8.83 & $9.61 \mathrm{E}-07$ \\
\hline TOMM7 & Mitochondrial import receptor subunit TOM7 & 8.16 & 0.025 \\
\hline ATP5B & ATP synthase subunit beta, mitochondrial & 7.76 & $1.81 \mathrm{E}-05$ \\
\hline UQCRC2 & Cytochrome b-c1 complex subunit 2, mitochondrial & 6.75 & 2.34E-05 \\
\hline FASN & Fatty acid synthase & 6.60 & 0.0002 \\
\hline $\mathrm{IDH} 2$ & Isocitrate dehydrogenase [NADP] 2 & 5.77 & $6.95 \mathrm{E}-06$ \\
\hline COX5A & Cytochrome $c$ oxidase subunit $5 \mathrm{~A}$, mitochondrial & 4.99 & 6.99E-06 \\
\hline $\mathrm{ECH} 1$ & Delta(3,5)-Delta(2,4)-dienoyl-CoA isomerase, mitochondrial & 4.95 & 0.0006 \\
\hline MCCC2 & Methylcrotonoyl-CoA carboxylase beta chain, mitochondrial & 4.84 & $2.55 \mathrm{E}-05$ \\
\hline GATC & Glutamyl-tRNA(GIn) amidotransferase subunit $C$, mitochondrial & 4.32 & 9.33E-06 \\
\hline ETFA & Electron transfer flavoprotein subunit alpha, mitochondrial & 4.26 & 7.67E-05 \\
\hline $\mathrm{HADH} 2$ & HSD17B10/HADH2; 3-hydroxyacyl-CoA dehydrogenase & 4.26 & 0.0002 \\
\hline ACADVL & Very-long-chain specific acyl-CoA dehydrogenase, mitochondrial & 4.06 & 0.0001 \\
\hline AK2 & Adenylate kinase 2 , mitochondrial & 4.00 & 0.0001 \\
\hline IDH3A & Isocitrate dehydrogenase [NAD] subunit alpha, mitochondrial & 3.83 & $5.05 \mathrm{E}-05$ \\
\hline HADHA & Trifunctional enzyme subunit alpha, mitochondrial & 3.43 & 2.89E-05 \\
\hline CS & Citrate synthase, mitochondrial & 3.40 & 7.87E-06 \\
\hline HSPD1 & $60 \mathrm{kDa}$ heat shock protein, mitochondrial & 3.30 & 2.36E-05 \\
\hline DECR1 & 2,4-dienoyl-CoA reductase, mitochondrial & 3.29 & 0.001 \\
\hline SLC25A10 & Mitochondrial dicarboxylate carrier & 3.24 & 0.0001 \\
\hline GSR & Glutathione reductase, mitochondrial & 3.19 & 0.01 \\
\hline CYC1 & Cytochrome c1, heme protein, mitochondrial & 2.91 & 3.89E-06 \\
\hline NDUFA5 & NADH dehydrogenase [ubiquinone] 1 alpha subcomplex subunit 5 & 2.83 & 5.39E-05 \\
\hline PRDX5 & Peroxiredoxin-5, mitochondrial & 2.62 & 0.0009 \\
\hline PRKDC & DNA-dependent protein kinase, catalytic subunit (maintains mt-DNA) & 2.25 & 0.008 \\
\hline UQCR10 & Cytochrome b-c1 complex subunit 9 & 2.22 & 0.037 \\
\hline SDHA & Succinate dehydrogenase complex, subunit $A$, flavoprotein & 2.20 & 0.001 \\
\hline ABAT & 4-aminobutyrate aminotransferase, mitochondrial & 2.14 & 0.003 \\
\hline CYCS & Cytochrome c, somatic & 2.13 & $9.35 \mathrm{E}-05$ \\
\hline MRPL15 & Mitochondrial ribosomal protein L15 & 2.11 & 0.002 \\
\hline \multicolumn{4}{|c|}{ Glycolysis and Pentose Phosphate Pathway (PPP) Related Enzymes (17) } \\
\hline GAPDH & Glyceraldehyde-3-phosphate dehydrogenase & 25.19 & 1.13E-05 \\
\hline PGAM1 & Phosphoglycerate mutase 1 (Brain) & 16.82 & 6.97E-06 \\
\hline G6PD & Glucose-6-phosphate 1-dehydrogenase & 9.29 & 1.37E-06 \\
\hline ENO1 & Alpha-enolase & 9.08 & $1.08 \mathrm{E}-05$ \\
\hline PKM2 & Pyruvate kinase & 8.15 & 0.0016 \\
\hline ALDOA & Fructose-bisphosphate aldolase & 6.87 & $3.53 E-06$ \\
\hline HK2 & Hexokinase-2 & 5.71 & 2.43E-06 \\
\hline GPI & Glucose-6-phosphate isomerase & 5.61 & 4.43E-05 \\
\hline TALD01 & Transaldolase & 5.20 & 0.00095 \\
\hline LDHA & L-lactate dehydrogenase A & 4.65 & 2.82E-05 \\
\hline PGD & 6-phosphogluconate dehydrogenase, decarboxylating & 4.21 & 3.24E-05 \\
\hline PGK1 & Phosphoglycerate kinase 1 & 3.97 & 0.01 \\
\hline HK3 & Hexokinase-3 & 3.66 & 0.0003 \\
\hline PFKP & 6-phosphofructokinase type C & 3.48 & 0.0002 \\
\hline PGAM2 & Phosphoglycerate mutase 2 & 3.38 & 0.006 \\
\hline FBP1 & Fructose-1,6-bisphosphatase 1 & 2.72 & 0.003 \\
\hline TKT & Transketolase & 2.15 & 0.0003 \\
\hline
\end{tabular}

\section{Proteomics analysis of MCF7-hTERT-eGFP cells}

In order to dissect the mechanism(s) by which high telomerase activity drives the survival and clonal expansion of CSCs, we next employed an unbiased labelfree proteomics approach. The proteome of fractionated MCF7-hTERT-eGFP cells was directly determined, after FACS separation into GFP-high and GFP-low populations.
For simplicity, we focused on the proteins that were overexpressed in GFP-high cells by $>1.5$-fold. Our results are summarized in Tables 1, 2 and 3.

Importantly, $>30$ mitochondrial-related proteins were over-expressed in GFP-high cells (Table 1). Most of these proteins were related to oxidative phosphorylation, the TCA cycle, or mitochondrial biogenesis. Remarkably, MT-CO2 (a mt-DNA encoded protein) was upregulated by $>20$-fold, reflecting a significant increase in mitochondrial 
Table 2. Key Molecules Up-regulated in hTERT-GFP(+) MCF7 Cells Isolated by FACS: the EMT.

Symbol Description

Fold-Upregulation

$(\mathrm{GPF}(+) / \mathrm{GFP}(-))$

ANOVA

EMT-Related Marker Proteins (17)

MTPN Myotrophin 41.58

0.0016

KRT19 Keratin, type I cytoskeletal 19 15.04

ACTR3 Actin-related protein $3 \quad 12.74$

$\begin{array}{llr}\text { FLNB } & \text { Filamin-B } & 9.30\end{array}$

GSN Gelsolin $\quad 9.07$

ACTN4 Alpha-actinin-4 $\quad 6.63$

ACTA2 Actin, aortic smooth muscle

ACTN1 Alpha-actinin-1 $\quad 5.65$

CKAP4 Cytoskeleton-associated protein 4

MYO5C Unconventional myosin-Vc 3.84

SPTAN1 Spectrin alpha chain, non-erythrocytic 1

TAGLN2 Transgelin-2 $\quad 3.55$

CD44 CD44 antigen $\quad 3.20$

$\begin{array}{llr}\text { MYOF } & \text { Myoferlin } & 2.47\end{array}$

MYH14 Myosin-14 2.24

PFN2 Profilin 2.21

SPTBN1 Spectrin beta non-erythrocytic 1

\section{Markers of Proliferation (3)}

2.45E-05

MCM3 MCM3 mini-chromosome maintenance deficient 3

PA2G4

Proliferation-associated protein $2 \mathrm{G} 4$

Anti-Oxidant Proteins (2)

NQO1 NAD(P)H dehydrogenase, quinone $1 \quad 6.43$

SOD1 Superoxide dismutase [Cu-Zn] 4.49

3.90E-05

2.13E-07

A

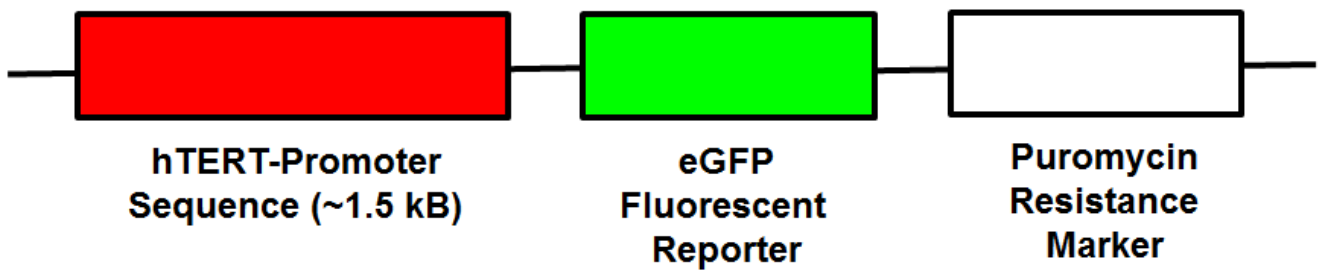

B

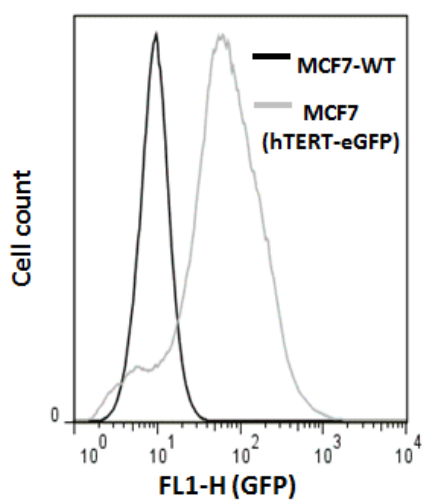

C

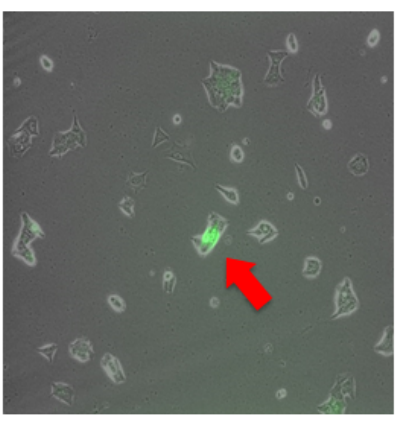

D

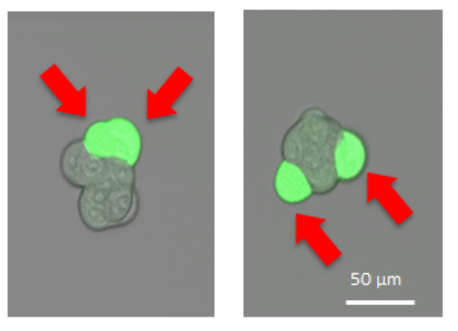

Figure 2: Generation of MCF7 cells harboring the hTERT-eGFP reporter, to select a sub-population of cells with high telomerase activity. Panel A. MCF7 cells were transduced with a lentiviral vector driving eGFP protein expression, under the control of a 1.5-kB fragment of the hTERT promoter. This DNA construct also contains a puromycin-resistance cassette for antibioticresistance selection. Panel B. After selection with puromycin, MCF7-hTERT-eGFP cells were subjected to FACS sorting to visualize the broad distribution of eGFP expression, which serves as a surrogate marker of telomerase activity. Panel C. Note that very few cells in MCF7-hTERT-eGFP monolayers showed high GFP fluorescence. Panel D. Note the dramatic enrichment of GFP $(+)$ cells in MCF7 cell mammospheres (>50 $\mu \mathrm{m})$, each containing usually 1-to-2 GFP-high cells. 
biogenesis. Consistent with an overall anabolic phenotype, 17 enzymes related to glycolysis and the pentose phosphate pathway were also upregulated in GFP-high cells (Table 1).

CSCs undergo an EMT, which facilitates cell migration, invasion and metastatic dissemination [8]. Thus, we also examined the expression of EMT markers, and cytoskeletal proteins associated with cell migration (Table 2). Greater than 17 proteins known to be associated with this EMT phenotype were upregulated in GFP-high cells. Such examples include myotrophin ( $>40$-fold), keratin-19 (>15-fold), smooth muscle actin $(>5$-fold), and CD44 (>3-fold). Markers of proliferation (PCNA; >6-

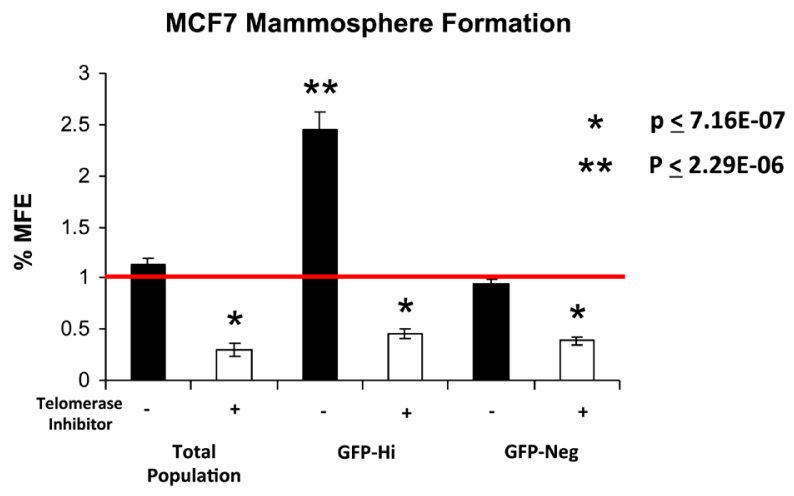

Figure 3: hTERT-eGFP-high MCF7 cells form mammospheres more efficiently, in a telomerasedependent manner. MCF7-hTERT-eGFP cells were fractionated into GFP-high (top 5\%) and GFP-low (negative) groups. Then, five thousands cells from each group were seeded per well in 6-well low-attachment plates. These two groups were also compared to the total unfractionated cell population. Note that GFP-high cells form mammospheres with an efficiency nearly 2.5 -fold greater than GFP-negative cells and the total unfractionated cell population. In this context, mammosphere formation was sensitive to that administration of a well-characterized telomerase inhibitor, namely MST-312, as expected. MFE, mammosphere forming efficiency.
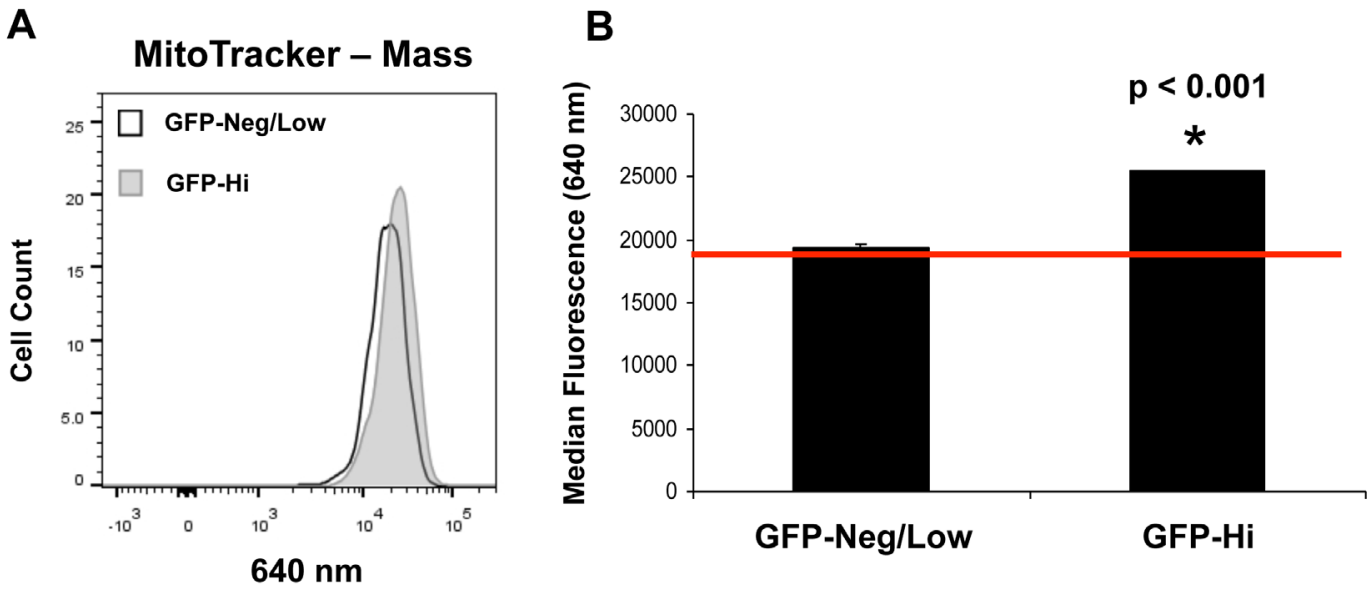

Figure 4: hTERT-eGFP-high MCF7 cells show an increase in mitochondrial mass. Panel A. Note that as compared to GFPlow cells (bottom 5\%), GFP-high cells (top 5\%) demonstrate a significant shift to the right, for mitochondrial mass (MitoTracker Deep-Red probe). Panel B. FACS quantification of median fluorescence intensity is presented. As such, increased mitochondrial mass (1.3-fold) may be a critical element of the anabolic TIC phenotype. $p<0.001$. fold), and the anti-oxidant response (NQO1; >6-fold) were also significantly increased.

Elevated protein synthesis is another important feature of the anabolic CSC phenotype [9]. As predicted, GFP-high cells show the upregulation of $>30$ proteins related to protein synthesis (Table 3), including protein folding chaperones, ribosome-related proteins, translation initiation factors, peptide elongation factors, as well as enzymes for tRNA synthesis.

Taken together, hTERT-eGFP-high cells overexpress $>100$ proteins related to an anabolic CSC phenotype.

\section{Relevance of hTERT targets in human breast cancers cells in vivo}

To determine the clinical relevance of our findings, we next assessed whether the hTERT proteomic targets that we identified in GFP-high cells were transcriptionally over-expressed, in human breast cancer cells in vivo. For this purpose, we exploited a clinical data set of tumor samples from $N=28$ breast cancer patients. These tumor samples were subjected to laser-capture micro-dissection, to separate epithelial cancer cells from adjacent tumor stroma [10]. Overall, greater than seventy hTERT targets (related to mitochondria, glycolysis, the EMT, and protein synthesis) that we identified in GFP-high cells were also transcriptionally elevated in human breast cancer cells in vivo. Tables 4, 5 and 6 present a detailed summary of these findings. These new hTERT protein targets that we identified in MCF7-hTERT-eGFP cells may be especially relevant for improving human breast cancer diagnosis and therapy. 
Table 3. Key Molecules Up-regulated in hTERT-GFP(+) MCF7 Cells Isolated by FACS: Protein Synthesis and Chaperones.

\section{Symbol Description}

Chaperones for Protein Folding (10)

PPIA Peptidyl-prolyl cis-trans isomerase A

HSPA2 Heat shock-related 70kDa protein 2

HSPA1L Heat shock $70 \mathrm{kDa}$ protein 1-like

HSPA1B Heat shock $70 \mathrm{kDa}$ protein $1 \mathrm{~A}$

HSP90AB1 Heat shock protein HSP 90-beta

HSP90B1 Heat shock protein Grp94

HSPA8 Heat shock cognate $71 \mathrm{kDa}$ protein

HSPD1 $60 \mathrm{kDa}$ heat shock protein, mitochondrial

HSPA4

FKBP4
Fold-Upregulation (GPF(+)/GFP(-))

Infinity

27.53

10.66

9.65

6.37

6.13

4.40

3.30

2.90

2.21

6.70

4.78

4.16

3.55

3.18

2.91

2.53

2.28

2.03

2.00

6.12

3.36

2.17

2.06

1.88

14.43

4.77

2.53

2.18
ANOVA

0.007

0.003

0.01

0.0007

5.08E-06

0.036

0.0003

2.36E-05

4.57E-05

0.003

1.08E-05

3.87E-06

0.00075

0.0001

0.0001

0.00016

0.0002

0.004

0.0008

0.018

0.00016

0.0002

0.0009

0.001

0.003

5.54E-07

$1.42 \mathrm{E}-05$

0.0003

0.001

EEF1B2 Elongation factor 1-beta

\section{A}

\section{MitoTracker - Activity}

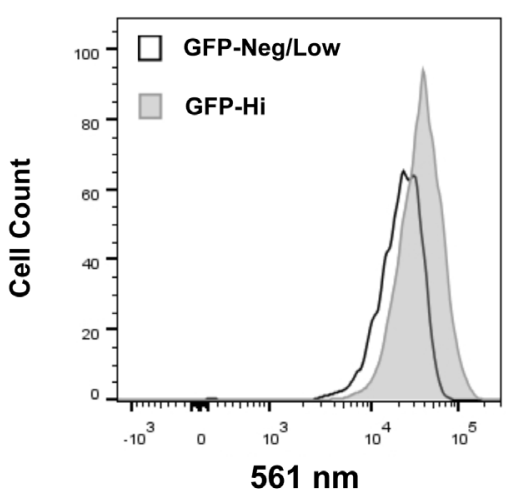

B

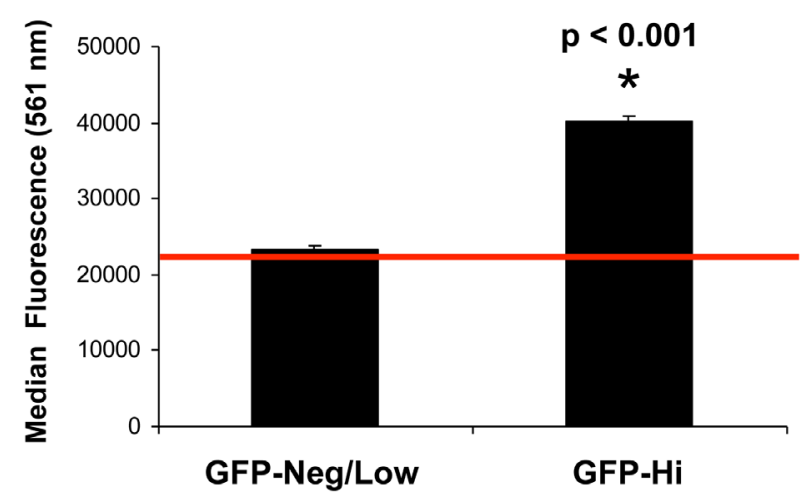

Figure 5: hTERT-eGFP-high MCF7 cells show an increase in mitochondrial activity. Panel A. Note that as compared to GFP-low cells (bottom 5\%), GFP-high cells (top 5\%) demonstrate a significant shift to the right, for mitochondrial membrane potential (MitoTracker Orange probe). Panel B: FACS quantification of median fluorescence intensity is presented, representing a 1.7-fold increase. $p<0.001$. 
Table 4. hTERT Protein Targets Transcriptionally Up-regulated in Breast Cancer: Focus on Mitochondria and Glycolysis.

\begin{tabular}{|c|c|c|c|}
\hline Symbol & Gene Description & $\begin{array}{l}\text { Up-regulation } \\
\text { (fold-change) }\end{array}$ & P-value \\
\hline \multicolumn{4}{|c|}{ Mitochondrial-Related Proteins (23) } \\
\hline$\overline{\mathrm{MCCC} 2}$ & Methylcrotonoyl-CoA carboxylase beta chain, mitochondrial & 5.48 & $5.78 \mathrm{E}-07$ \\
\hline ATP5B & ATP synthase subunit beta, mitochondrial & 5.04 & $2.75 \mathrm{E}-06$ \\
\hline UQCRC2 & Cytochrome b-c1 complex subunit 2 , mitochondrial & 4.84 & $5.73 \mathrm{E}-06$ \\
\hline PARK7 & Protein DJ-1 & 4.26 & 4.08E-05 \\
\hline ECHS1 & Enoyl-CoA hydratase, mitochondrial & 4.05 & $8.22 \mathrm{E}-05$ \\
\hline $\mathrm{COX} 5 \mathrm{~A}$ & Cytochrome c oxidase subunit $5 \mathrm{~A}$, mitochondrial & 3.62 & $3.22 \mathrm{E}-04$ \\
\hline HSPD1 & $60 \mathrm{kDa}$ heat shock protein, mitochondrial & 3.42 & 5.93E-04 \\
\hline DECR1 & 2,4-dienoyl-CoA reductase, mitochondrial & 3.38 & $6.86 \mathrm{E}-04$ \\
\hline HADHA & Trifunctional enzyme subunit alpha, mitochondrial & 3.27 & 9.34E-04 \\
\hline CYC1 & Cytochrome c1, heme protein, mitochondrial & 3.08 & 1.64E-03 \\
\hline TOMM7 & Mitochondrial import receptor subunit TOM7 & 3.03 & $1.85 \mathrm{E}-03$ \\
\hline CYCS & Cytochrome c, somatic & 2.92 & $2.52 \mathrm{E}-03$ \\
\hline NDUFA5 & NADH dehydrogenase [ubiquinone] 1 alpha subcomplex subunit 5 & 2.75 & 4.07E-03 \\
\hline CS & Citrate synthase, mitochondrial & 2.66 & $5.13 \mathrm{E}-03$ \\
\hline IDH2 & Isocitrate dehydrogenase [NADP] 2 & 2.46 & 8.55E-03 \\
\hline GRPEL1 & GrpE protein homolog 1, mitochondrial & 2.39 & $1.01 \mathrm{E}-02$ \\
\hline MRPL15 & Mitochondrial ribosomal protein L15 & 2.26 & $1.39 \mathrm{E}-02$ \\
\hline AK2 & Adenylate kinase 2 , mitochondrial & 2.20 & $1.59 \mathrm{E}-02$ \\
\hline IDH3A & Isocitrate dehydrogenase [NAD] subunit alpha, mitochondrial & 2.16 & $1.78 \mathrm{E}-02$ \\
\hline PRKDC & DNA-dependent protein kinase, catalytic subunit (maintains mt-DNA) & 2.14 & $1.85 \mathrm{E}-02$ \\
\hline ABAT & 4-aminobutyrate aminotransferase, mitochondrial & 2.08 & 2.14E-02 \\
\hline $\mathrm{ECH} 1$ & Delta(3,5)-Delta(2,4)-dienoyl-CoA isomerase, mitochondrial & 1.97 & $2.72 \mathrm{E}-02$ \\
\hline ETFA & Electron transfer flavoprotein subunit alpha, mitochondrial & 1.75 & 4.25E-02 \\
\hline \multicolumn{4}{|c|}{ Glycolysis and Pentose Phosphate Pathway (PPP) Related Enzymes (11) } \\
\hline TALD01 & Transaldolase & 4.13 & $6.35 \mathrm{E}-05$ \\
\hline ALDOA & Fructose-bisphosphate aldolase & 3.60 & $3.45 \mathrm{E}-04$ \\
\hline GPI & Glucose-6-phosphate isomerase & 3.36 & 7.28E-04 \\
\hline FBP1 & Fructose-1,6-bisphosphatase 1 & 3.35 & 7.47E-04 \\
\hline PKM2 & Pyruvate kinase & 3.26 & $9.79 E-04$ \\
\hline GAPDH & Glyceraldehyde-3-phosphate dehydrogenase & 2.97 & 2.22E-03 \\
\hline PGK1 & Phosphoglycerate kinase 1 & 2.46 & 8.66E-03 \\
\hline PGAM1 & Phosphoglycerate mutase 1 (Brain) & 2.55 & 6.87E-03 \\
\hline LDHA & L-lactate dehydrogenase A & 2.42 & $9.42 \mathrm{E}-03$ \\
\hline TKT & Transketolase & 2.20 & $1.60 \mathrm{E}-02$ \\
\hline ENO1 & Alpha-enolase & 1.96 & $2.75 \mathrm{E}-02$ \\
\hline
\end{tabular}

-Transcriptional profiling data derived from the analysis of $\mathrm{N}=\mathbf{2 8}$ breast cancer patients are shown, high-lighting the levels of foldupregulation observed in the epithelial cancer cell compartment (relative to the tumor stroma), and corresponding $p$-values derived from the analysis of these clinical samples.

\section{hTERT-eGFP-high MCF7 cells show an increase in mitochondrial mass and functional activity}

To directly validate the mitochondrial phenotype of hTERT-eGFP-high cells (top 5\%), we used two wellestablished fluorescent probes to quantitate mitochondrial membrane potential and mass, by FACS analysis. More specifically, we used MitoTracker Orange (561-nm) as a reporter for mitochondrial membrane potential and MitoTracker Deep-Red (640-nm) as a marker of mitochondrial mass. Importantly, Figures 4 and 5 show that as compared to GFP-low cells (bottom 5\%), GFP-high cells (top 5\%) demonstrate a significant shift to the right, for both mass and membrane potential. FACS quantitation of median fluorescence intensity shows that both of these mitochondrial-related measurements are significantly elevated in GFP-high cells. As such, mitochondrial mass and function may be critical elements of the anabolic TIC phenotype.

\section{Using large cell size to enrich telomerase activity and mitochondrial mass}

Previous studies using mouse mammary epithelial cells have demonstrated that stem-like cells can be enriched solely based on cell size [11]. For example, large stem-like cells with diameters $>10 \mu \mathrm{m}$, defined by higher forward scatter during FACS analysis, showed a $>4$-fold increased ability to undergo 3-D mammosphere formation. Moreover, these large stem-like mammary cells also had the ability to efficiently repopulate and regenerate the mammary gland in vivo [11].

Thus, here we fractionated MCF7-hTERT-eGFP cells by size, based on forward/side scatter, into two populations: i) larger cells ( $\sim 15 \%$ of the total population) and ii) smaller cells ( $\sim 85 \%$ of the total population) (Figure 6). Interestingly, larger MCF7 cells showed a 2.65 -fold increase in hTERT-eGFP fluorescence, as compared with the smaller cell population. Importantly, larger 
Table 5. hTERT Protein Targets Transcriptionally Up-regulated in Breast Cancer: Focus on the EMT.

\begin{tabular}{|c|c|c|c|}
\hline Symbol & Gene Description & $\begin{array}{l}\text { Up-regulation } \\
\text { (fold-change) }\end{array}$ & P-value \\
\hline \multicolumn{4}{|c|}{ EMT-Related Marker Proteins (11) } \\
\hline FLNB & Filamin-B & 4.81 & $6.21 \mathrm{E}-06$ \\
\hline KRT19 & Keratin, type I cytoskeletal 19 & 4.39 & 2.66E-05 \\
\hline SPTAN1 & Spectrin alpha chain, non-erythrocytic 1 & 4.19 & $5.16 \mathrm{E}-05$ \\
\hline MYO5C & Unconventional myosin- $\mathrm{Vc}_{\mathrm{c}}$ & 3.79 & $1.90 \mathrm{E}-04$ \\
\hline CD44 & CD44 antigen & 3.44 & 5.69E-04 \\
\hline ACTR3 & Actin-related protein 3 & 3.15 & 1.35E-03 \\
\hline MYOF & Myoferlin & 2.67 & 5.00E-03 \\
\hline TAGLN2 & Transgelin-2 & 2.42 & 9.47E-03 \\
\hline PFN2 & Profilin & 2.16 & $1.78 \mathrm{E}-02$ \\
\hline ACTN4 & Alpha-actinin-4 & 2.12 & 1.94E-02 \\
\hline CKAP4 & Cytoskeleton-associated protein 4 & 1.88 & $3.29 \mathrm{E}-02$ \\
\hline \multicolumn{4}{|c|}{ Markers of Proliferation (1) } \\
\hline PCNA & Proliferating cell nuclear antigen & 3.58 & 3.64E-04 \\
\hline \multicolumn{4}{|c|}{ Anti-Oxidant Proteins (2) } \\
\hline$\overline{\text { SOD1 }}$ & Superoxide dismutase [Cu-Zn] & 5.37 & 8.58E-07 \\
\hline NQ01 & $\mathrm{NAD}(\mathrm{P}) \mathrm{H}$ dehydrogenase, quinone 1 & 3.49 & 4.81E-04 \\
\hline
\end{tabular}

-Transcriptional profiling data derived from the analysis of $\mathrm{N}=\mathbf{2 8}$ breast cancer patients are shown, high-lighting the levels of foldupregulation observed in the epithelial cancer cell compartment (relative to the tumor stroma), and corresponding $\mathrm{p}$-values derived from the analysis of these clinical samples.

Table 6. hTERT Protein Targets Transcriptionally Up-regulated in Breast Cancer: Focus on Protein Synthesis and Chaperones.

\begin{tabular}{|c|c|c|c|}
\hline Symbol & Gene Description & $\begin{array}{l}\text { Up-regulation } \\
\text { (fold-change) }\end{array}$ & P-value \\
\hline \multicolumn{4}{|c|}{ Chaperones for Protein Folding (8) } \\
\hline FKBP4 & Peptidyl-prolyl cis-trans isomerase & 5.02 & 2.95E-06 \\
\hline HSP90AB1 & Heat shock protein HSP 90-beta & 4.93 & 4.03E-06 \\
\hline PPIA & Peptidyl-prolyl cis-trans isomerase A & 4.29 & $3.74 \mathrm{E}-05$ \\
\hline HSPA4 & Heat shock $70 \mathrm{kDa}$ protein 4 & 3.75 & 2.18E-04 \\
\hline HSPD1 & $60 \mathrm{kDa}$ heat shock protein, mitochondrial & 3.42 & 5.93E-04 \\
\hline HSPA8 & Heat shock cognate 71 kDa protein & 2.54 & 7.06E-03 \\
\hline HSP90B1 & Heat shock protein Grp94 & 2.43 & $9.33 \mathrm{E}-03$ \\
\hline HSPA1B & Heat shock $70 \mathrm{kDa}$ protein $1 \mathrm{~A}$ & 1.56 & $6.29 E-02$ \\
\hline \multicolumn{4}{|c|}{ Ribosomal Proteins (10) } \\
\hline RPL7 & $60 S$ ribosomal protein $\mathrm{L} 7$ & 5.21 & 1.53E-06 \\
\hline RPS2 & $40 S$ ribosomal protein $S 2$ & 4.77 & 7.21E-06 \\
\hline RPL15 & $60 S$ ribosomal protein $\mathrm{L} 15$ & 4.60 & $1.28 \mathrm{E}-05$ \\
\hline RPS3A & $40 S$ ribosomal protein S3A & 4.59 & 1.35E-05 \\
\hline RPS5 & $40 S$ ribosomal protein S5 & 4.41 & 2.45E-05 \\
\hline RPL32 & $60 S$ ribosomal protein L32 & 4.03 & 8.74E-05 \\
\hline RPS4X & $40 S$ ribosomal protein $S 4, X$-linked isoform & 3.92 & 1.27E-04 \\
\hline RPS15 & $40 S$ ribosomal protein $\mathrm{S} 15$ & 3.72 & $2.41 \mathrm{E}-04$ \\
\hline RPL11 & $60 S$ ribosomal protein L11 & 2.74 & 4.16E-03 \\
\hline \multicolumn{4}{|c|}{ Translation initiation factors (2) } \\
\hline EIF3F & Eukaryotic translation initiation factor 3 subunit $F$ & 5.07 & 2.48E-06 \\
\hline EIF4A2 & Eukaryotic initiation factor 4A-II & 2.68 & $4.83 \mathrm{E}-03$ \\
\hline \multicolumn{4}{|c|}{ Elongation factors (4) } \\
\hline EEF1B2 & Elongation factor 1 -beta & 4.08 & 7.56E-05 \\
\hline EEF2 & Elongation factor 2 & 4.01 & $9.29 \mathrm{E}-05$ \\
\hline EEF1G & Elongation factor 1-gamma & 3.71 & 2.44E-04 \\
\hline EEF1A1 & Elongation factor 1 alpha 1 & 3.16 & 1.30E-03 \\
\hline \multicolumn{4}{|c|}{ Enzymes for tRNA synthesis (5) } \\
\hline EPRS & Bifunctional glutamate/proline--tRNA ligase & 4.06 & 8.10E-05 \\
\hline KARS & Lysine--tRNA ligase & 2.81 & $3.49 \mathrm{E}-03$ \\
\hline
\end{tabular}

-Transcriptional profiling data derived from the analysis of $\mathrm{N}=28$ breast cancer patients are shown, high-lighting the levels of foldupregulation observed in the epithelial cancer cell compartment (relative to the tumor stroma), and corresponding $p$-values derived from the analysis of these clinical samples. 
cells also showed a 1.6-fold increase in mitochondrial mass (MitoTracker Deep-Red) and a 2.4-fold increase in mitochondrial activity (membrane potential), as measured using MitoTracker Orange (Figure 6).

As such, larger cell size in MCF7 cells directly correlates with telomerase activity (cell immortalization) and mitochondrial mass/activity, which would be consistent with an anabolic CSC phenotype. These results provide independent validation for the idea that high hTERT activity ("stemness") is functionally associated with increased mitochondrial mass and activity in breast cancer cells, and co-segregates with large cell size. Importantly, large cell size is determined by increased $\mathrm{PI} 3 \mathrm{~K} / \mathrm{AKT} / \mathrm{mTOR}$-signaling, which drives significant increases in overall protein synthesis [12-14]. This finding is consistent with our results from proteomics analysis, showing an increase in the abundance of the protein synthesis machinery (See Tables 3 and 6).

\section{DISCUSSION}

Here, we have used an hTERT-promoter-eGFPreporter system to identify and purify a sub-population of MCF7 cells, with high hTERT transcriptional activity, by FACS analysis. These hTERT-eGFP-high cells formed mammospheres with greater efficiency, as predicted, consistent with the idea that this sub-population of cells is enriched in cancer stem-like cells. Importantly, proteomics analysis of these hTERT-eGFP-high MCF7 cells revealed the upregulation of mitochondrial proteins, glycolytic enzymes and EMT markers, as well

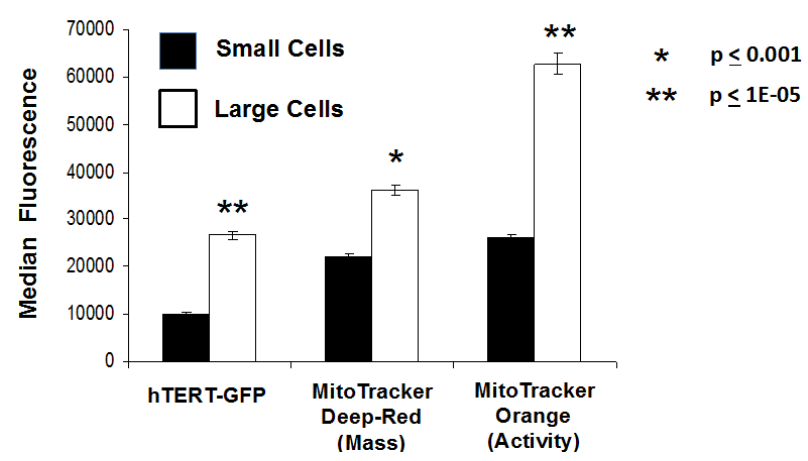

Figure 6: Fractionation of hTERT-eGFP MCF7 cells by cell size allows the separation of larger and smaller cell sub-populations, with distinct metabolic functional properties. We fractionated MCF7-hTERT-eGFP cells based on forward/side scatter into larger and smaller cell populations. Note that larger MCF7 cells showed a 2.65 -fold increase in hTERT-eGFP fluorescence, as compared with the smaller cell population. Similarly, larger cells also showed a 1.6fold increase in mitochondrial mass (MitoTracker Deep-Red) and a 2.4-fold increase in mitochondrial activity (membrane potential), as measured using MitoTracker Orange. Thus, larger cell size directly correlates with telomerase activity and mitochondrial mass/activity, which would be consistent with an anabolic CSC phenotype. as components of the protein synthesis machinery, such as ribosome-related proteins and chaperones for protein folding. Interestingly, MT-CO2 (cytochrome c oxidase subunit 2; Complex IV) expression was increased by $>20$ fold. As MT-CO2 is encoded by mt-DNA, this finding is indicative of increased mitochondrial biogenesis in hTERT-eGFP-high MCF7 cells. We then functionally validated that hTERT-eGFP-high MCF7 cells show increases in mitochondrial mass and activity, using two distinct MitoTracker probes. Complementary results were obtained using cell size to fractionate MCF7 cells. Larger stem-like cells showed increased hTERT-GFP levels, as well as increased mitochondrial mass and function. Thus, these two independent approaches for the enrichment of immortal anabolic CSCs should allow the development of new prognostic biomarkers and related novel anti-cancer

Understanding Tumor Metabolic Heterogeneity

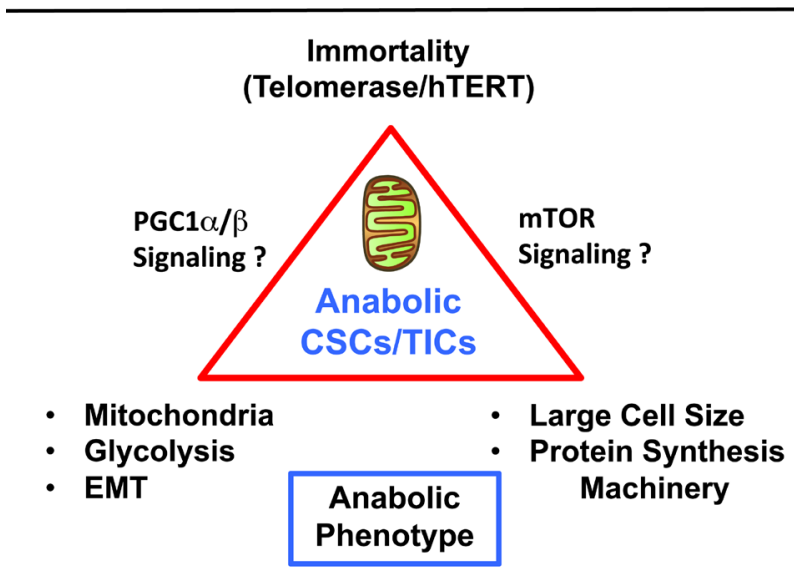

Figure 7: Understanding the role of immortality, anabolic metabolism and cell size in stem-like cancer cells. Here, we used FACS analysis to begin to dissect metabolic heterogeneity in tumor cells. More specifically, we showed that anabolic stem-like cancer cells can be purified using hTERTeGFP as a surrogate marker of telomerase activity. These eGFPhigh cells showed increased stem cell activity (mammosphere formation), as well as functional increases in mitochondrial mass and activity. Further studies with unbiased label-free proteomics revealed the upregulation of mitochondrial proteins, glycolytic enzymes and EMT markers, as well as ribosome subunits and other components of the proteins synthesis machinery. These proteomic studies are consistent with, and support an anabolic stem-like cancer cell phenotype. Quantitatively similar results were obtained using large cell size to purify anabolic stem-like cancer cells. We speculate that high telomerase activity drives an increase in mitochondrial power, via positive regulation by PGC $1-\alpha / \beta$. Moreover, we suggest that increased mTOR signaling may contribute to larger cell size, via increased protein synthesis. The PI3K/AKT/mTOR pathway is known to i) control cell size by positively regulating protein synthesis and ii) telomerase (hTERT) forms a physical complex with mTOR and can therefore regulate its activity. In summary, our results metabolically define a sub-population of stem-like, mitochondrial-rich, cancer cells, allowing us to understand the possible origins of metabolic heterogeneity in human tumors. 
therapies.

Interestingly, recent studies in aging and cancer have both directly linked telomerase activity to mitochondrial function, via the hTERT-p53-PGC1 signaling axis [15-18]. More specifically, telomerase is required for the proper expression of PGC1- $\alpha / \beta$, a major nuclearencoded mitochondrial transcription factor. Studies in aging demonstrate that a loss of telomerase functionally impairs mitochondria, via the induction of p53 which down-regulates PGC1- $\alpha / \beta$ expression [16]. Conversely, during tumorigenesis, high telomerase expression helps to promote cancer progression, by reducing p53 and, therefore, elevating PGC1- $\alpha / \beta$ expression [1921]. Taken together, these mechanistic studies are consistent with our current findings, in which we link telomerase transcriptional activity in cancer stem cells to mitochondrial biogenesis, both functionally and via proteomics analysis (Figure 7).

Cell size is normally controlled by the PI3K/AKT/ mTOR-pathway, which converges on protein synthesis, via S6-kinase signaling [12]. Therefore, large cell size is known to be associated with increased protein synthesis $[13,14]$. Interestingly, telomerase (hTERT) forms a physical complex with mTOR and can therefore regulate its functional activity $[22,23]$. Consistent with this hypothesis, we demonstrate here that stem-like anabolic cancer cells, with high-telomerase transcriptional activity, also show remarkable increases in i) protein synthesis machinery (chaperones, ribosome-related proteins, translation initiation factors, peptide elongation factors, enzymes for tRNA synthesis) and ii) are associated with large cell size (Figure 7).

Interestingly, large cell size is also a conserved characteristic of circulating tumor cells (CTCs), which share several properties with CSCs or TICs [24-26]. For example, CTCs - like CSCs - are found in clusters, are associated with distant metastasis, and can initiate tumor formation in xenografted mice [27]. Also, recent studies demonstrate that CTCs show an increase in mitochondrial mass and OXPHOS, most likely reflecting increased mitochondrial biogenesis, secondary to elevated levels of PGC1- $\alpha / \beta[28]$.

We recently directly compared the proteome of MCF7 cell monolayers to MCF7-derived mammospheres, using label-free unbiased proteomics analysis. Consistent with our current findings, this analysis demonstrated that MCF7-derived mammospheres show the overexpression of $>60$ mitochondrial-related proteins and $>80$ components of the protein synthesis machinery $[9,29]$. Complementary results were also obtained with MCF7 cells transfected with FOXM1, a key stem cell associated transcription factor that is a downstream target of the Wnt/ $\beta$-catenin signaling pathway [30]. Interestingly, MCF7FOXM1 cells showed a $>3$-fold increase in mammosphere formation, as compared with empty-vector alone control cells. Thus, several independent proteomic data sets now show the conserved up-regulation of both i) mitochondrial biogenesis and ii) protein synthesis, as key elements of the anabolic CSC phenotype.

Importantly, functional validation studies revealed that mammosphere formation could be efficiently blocked with well-established inhibitors of mitochondrial function and/or inhibitors of protein synthesis, such as oligomycin A and puromycin, respectively [9, 29]. Moreover, FDAapproved drugs which inhibit mitochondrial biogenesis or OXPHOS as a manageable side-effect (azithromycin, doxycycline and pyrvinium pamoate) also efficiently blocked mammosphere formation [31]; an FDA-approved inhibitor of protein synthesis, namely rapamycin, inhibited mammosphere formation as well [9]. Remarkably, the growth of mammosphere cultures derived from primary breast cancer cells (isolated from metastatic disease sites) was sensitive to inhibition with doxycycline [32]. Thus, these diverse classes of FDA-approved drugs should all be considered for testing in future phase II clinical trials, such as window-of-opportunity trials. In direct support of this idea, everolimus (Afinitor; a rapamycin-analogue and inhibitor of protein synthesis) has already shown significant efficacy in clinical trials in breast cancer patients [33].

In summary, we show here that high telomerase activity metabolically defines a sub-population of anabolic CSCs, with increased mitochondrial mass and large cell size. Overall, greater than seventy hTERT targets (related to mitochondria, glycolysis, the EMT, and protein synthesis) that we identified in hTERT-eGFP-high cells were also transcriptionally elevated in human breast cancer cells in vivo. Thus, the new hTERT metabolic targets that we identified here may be important for improving human breast cancer diagnosis and therapy.

\section{MATERIALS AND METHODS}

\section{Materials}

MCF7 cells were purchased from the ATCC. Gibcobrand cell culture media (DMEM and DMEM/F12) was purchased from Life Technologies. The lentiviral vector encoding the hTERT promoter linked to eGFP was custom-made by Genecopoeia (USA). MitoTracker probes (Deep Red and Orange) were purchased from Molecular Probes/Invitrogen, via Life Technologies. The telomerase inhibitor IX (MST-312; sc-204333) was obtained commercially from Santa Cruz Biotech (USA).

\section{Construction of the hTERT-eGFP lentiviral vector}

The following $1.5 \mathrm{kB}$ sequence was used as the hTERT-promoter to generate the hTERT-eGFP-Puro ${ }^{\mathrm{R}}$ cassette, and cloned into a lentiviral vector, custom-made 
by Genecopoeia:

5' - TAAAATTGTGTTTTCTATGTTG GCTTCTCTG C AGAGAACCAGTGTAAGCTACAACTTAACTTTTGTTGG AACAAATTTTCCAAACCGCCCCTTTGCCCTAGTGCA GAGACAATTCACAAACACAGCCCTTTAAAAAGGCTT AGGGATCACTAAGGGGATTTCTAGAAGAGCGACCTG TAATCCTAAGTATTTACAAGACGAGGCTAACCTCCAGG A GCGTGACAG CCCAGGGAGGGTGCGAG GCCTG TTCAAATGCTAGCTCCATAAATAAAGCAATTTCCTCC GGCAGTTTCTGAAAGTAGGAAAGGTTACATTTAA GGTTGCGTTTGTTAGCATTTCAGTGTTTGCCGACCT CAG CTACAG CATCCCTG CAAG GCTCG G GAGAC CCAGAAGTTTCTCGCCCCTTAGATCCAAACTTGAGC AACCCGGAGTCTGGATTCCTGGGAAGTCCTAGCTG TCCTG CG GTTGTG C C G G G CCCCA G TCTG G AGGGGACCAGTGGCCGTGTGGCTTCTACTGCTGGG CTGGAAGTCGGGCCTCCTAGCTCTGCAGTCCGAGGCTTGGAGCCAGGTGCCTGGACCCCGAG GTTGCCCTCCACCCTGTGCGGGCGGGATGTGACC AGATGTTGG CCTCATCTGCCAGACAGAGTGCCG G G G CCA G GTCAAG GCCGTTGTG GCTGGTG TGAGGCGCCCGGTGCGCGGCCAGCAGGAGCG CCTGGCTCCATTTCCCACCCTTTCTCGACGGGACC GCCCCGGTGGGTGATTAACAGATTTGGGGTGG TTTGCTCATGGTGGG GACCCCTCG CCG CCTG AGAACCTGCAAAGAGAAATGACGGGCCTGTG TCAAGGAGCCCAAGTCG CGGGGAAGTGTTGC AGGGAGGCACTCCGGGAGGTCCCGCGTGCCCGTCC AGGGAGCAATGCGTCCTCGGGTTCGTCCCCAGCCG CGTCTACGCGCCTCCGTCCTCCCCTTCACGTCCGGC ATTCGTGGTG CCCGGAGCCCGACG CCCCGCGTCCGGACCTGGAGGCAGCCCTGGGTCTCCGGATCAGGCCAGCG GCCAAAGGGTCGCCGCACGCACCTG TTCCCAGGGCCTCCACATCATGG CCCCTCCCTCG GGTTACCCCACAGCCTAGGCCGATTCGACCTCTCTCC GCTGGGGCCCTCGCTG GCGTCCCTGCACCCTGGG AGCGCGAGCGGCGCGCGGGCGGGGAAGCGCGG CCCAGACCCCCGGGTCCG CCCGGAGCAGCTGCG CTGTCGGGGCCAGGCCGGGCTCCCAGTGGATTCG CGGGCACAGACGCCCAGGACCGCGCTTCCCACG TGGCGGAGGGACTGGGGACCCGGGCACCCG TCCTGCCCCTTCACCTTCCAGCTCCGCCTCCTCC GCGCGGACCCCGCCCCGTCCCGACCCCTCCC G G G T C C C C G G C C CA G C C C C C T C C G G G C C TCCCAGCCCCTCCCCTTCCTTTCCGCGGCCCCG CCCTCTCCTCGCGGCGCGAGTT-3'.

\section{Viral transduction and cell selection}

Lentiviral particles harboring the hTERT-eGFPPuro $^{\mathrm{R}}$ cassette were prepared and used to stably transduce MCF7 cells, according to the manufacturer's protocol (in the presence of $5 \mu \mathrm{g} / \mathrm{ml}$ polybrene). Twenty-four hours post-infection, media containing the virus was removed and replaced with standard media. Cells were then selected with puromycin $(2 \mu \mathrm{g} / \mathrm{ml})$, for up to 10 days. Please note that for most of the experiments described in this paper, we compared the GFP-high (top 5\%) fraction to the GFP-low/negative (bottom 5\%) fraction, unless stated otherwise. However, for label-free proteomics analysis, we compared the GFP-high (top 10\%) population to the GFP-low (bottom 10\%) population, to insure that enough material was collected for sample processing. For these experiments, singlet FACS gating of live cells was utilized.

\section{Mammosphere formation}

A single cell suspension of MCF7-hTERT-eGFP cells was prepared using enzymatic (1x Trypsin-EDTA, Sigma Aldrich, \#T3924), and manual disaggregation (25 gauge needle) [34]. Cells were then plated at a density of 500 cells $/ \mathrm{cm}^{2}$ in mammosphere medium (DMEM-F12/ B27 / 20-ng/ml EGF/PenStrep) in nonadherent conditions, in culture dishes coated with (2-hydroxyethylmethacrylate) (poly-HEMA, Sigma, \#P3932). Cells were grown for 5 days and maintained in a humidified incubator at $37^{\circ} \mathrm{C}$ at an atmospheric pressure in $5 \%(\mathrm{v} / \mathrm{v})$ carbon dioxide/air. After 5 days for culture, spheres $>50 \mu \mathrm{m}$ were counted using an eye piece graticule, and the percentage of cells plated which formed spheres was calculated and is referred to as percentage mammosphere formation, and was normalized to one (1 $=100 \%$ MSE). Mammosphere assays were performed in triplicate and repeated three times independently.

\section{Treatment with telomerase inhibitor (MST-312)}

Cells were treated on day 0 with MST-312 at a concentration of $10 \mu \mathrm{M}$ and compared to vehicle alone controls, processed in parallel, after 5 days of mammosphere culture. A dose-response analysis of the effects of MST-312 on parental MCF7 cells was first performed to establish an effective concentration for the inhibition of mammosphere formation; our results showed that $1 \mu \mathrm{M}$ had little or no effect, while $10 \mu \mathrm{M}$ inhibited mammosphere formation by approximately $70 \%$ (data not shown). Therefore, experiments with eGFP fractionated MCF7 cells were carried out at $10 \mu \mathrm{M}$.

\section{Microscopy}

Fluorescent imaging of MCF7 cells in adherent conditions and non-adherent spheroid culture was performed using a Leica SP8 multi-photon DM6000 microscope to detect GFP expression and bright field images. 


\section{Proteomics analysis}

Cell lysates were prepared for trypsin digestion by sequential reduction of disulphide bonds with TCEP and alkylation with MMTS [35]. Then, the peptides were extracted and prepared for LC-MS/MS. All LC-MS/MS analyses were performed on an LTQ Orbitrap XL mass spectrometer (Thermo Scientific, San Jose, CA) coupled to an Ultimate 3000 RSLCnano system (Thermo Scientific, formerly Dionex, The Netherlands). Xcalibur raw data files acquired on the LTQ-Orbitrap XL were directly imported into Progenesis LCMS software (Waters Corp., Milford, MA, formerly Non-linear dynamics, Newcastle upon Tyne, UK) for peak detection and alignment. Data were analyzed using the Mascot search engine. Five technical replicates were analyzed for each sample type. Statistical analyses were performed using ANOVA and only fold-changes in proteins with a p-value less than 0.05 were considered significant.

\section{Bioinformatics analysis with human clinical samples}

To firmly establish the clinical relevance of our results from the quantitative proteomics, we re-analyzed the transcriptional profiles of epithelial breast cancer cells and adjacent tumor stromal cells that were physically separated by laser-capture microdissection (from $N=28$ human breast cancer patients) [10].

\section{FACS analysis}

For live cell sorting experiments, hTERT-eGFP transfected MCF7 cells were resuspended in PBS and sorted according to eGFP expression using the BD influx. Untransfected MCF7 cells were used as a negative control to determine positive GFP expression. Cells were sorted into two groups, those with the highest GFP expression and those with the lowest GFP expression. For FACs analysis experiments, hTERT-eGFP cells were labeled with MitoTracker Deep Red (\#M22426, Invitrogen) and MitoTracker Orange ((\#M7510, Invitrogen). Cells were kept of ice until analysis using the FACS Calibur. Results were analyzed using FlowJo software version 10. Cells were gated into two populations, those with the highest $5 \%$ of GFP expression, and those with the lowest 5\% and negative GFP expression. The median fluorescent intensity of MitoTracker Deep Red (640nm) and MitoTracker Orange $(561 \mathrm{~nm})$ was then determined within the two distinct GFP cellular populations. Additionally, cells were discriminated by cell size into two populations, small and large using SSC (side scatter) and FSC (forward scatter). The median intensity of eGFP, MitoTracker Deep Red, and MitoTracker Orange, within these populations, was then determined.

\section{Mitochondrial staining}

To measure mitochondrial activity, cells were stained with MitoTracker Orange (\#M7510, Invitrogen), whose accumulation in mitochondria is dependent upon membrane potential. To measure mitochondrial mass, cells were stained with MitoTracker Deep Red (\#M22426, Invitrogen), localizing to mitochondria regardless of mitochondrial membrane potential. Cells were incubated with pre-warmed MitoTracker staining solution (diluted in PBS/CM to a final concentration of $10 \mathrm{nM}$ ) for $30-60$ min at $37{ }^{\circ} \mathrm{C}$. All subsequent steps were performed in the dark. Cells were washed in PBS, harvested, and resuspended in $300 \mu \mathrm{L}$ of PBS. Cells were then analyzed by flow cytometry. Data analysis was performed using FlowJo software.

\section{Fractionation of MCF7 by cell size}

hTERT-eGFP-MCF7 cells, co-labeled with MitoTracker dyes, were analyzed by cell size using FlowJo software. Cells were separated by gating of forward scatter (FSC) and side scatter (SSC) plots into two populations for analysis, large cells ( $\sim 15 \%$ of the total population) and small cells ( $\sim 85 \%$ of the total population). The median fluorescent intensity of eGFP and MitoTracker dyes were determined in each population of cells. Very similar results were obtained, with either singlet FACS gating or all live cell FACS gating.

\section{Statistical analyses}

Statistical significance was determined using the Student's t-test or ANOVA, where appropriate. Values of less than 0.05 were considered significant. Data are shown as the mean \pm SEM, unless stated otherwise.

\section{ACKNOWLEDGMENTS}

We thank the University of Manchester for providing start-up funds that contributed to the success of this study. The Sotgia and Lisanti Laboratories were supported, in part, by funding from the European Union (ERC Advanced Grant), Breast Cancer Now, and the Manchester Cancer Research Centre (MCRC). Duncan L. Smith was core-funded by CRUK. Robert B. Clarke was funded by Breast Cancer Now. Ubaldo E. MartinezOutschoorn was supported by the National Cancer Institute (NCI) of the National Institutes of Health (NIH), under Award Number K08-CA175193-01A1. Richard G. Pestell (R.G.P.) was supported in part by grants from the NIH/NCI (RO1-CA70896, CA75503, CA86072), the 
Breast Cancer Research Foundation and the Department of Defense Concept Award W81XWH-1101-0303. The Sidney Kimmel Cancer Center was supported by the NIH Cancer Center Core Grant P30CA56036 (to R.G.P).

\section{Author Contributions}

MPL conceived and coordinated this UK and US joint project. RL performed all the experiments, analyzed the data and generated the figures with experimental data. MPL and FS wrote the first draft of the manuscript, which was extensively edited by BO, GB, DLS, RGP, UEM and RBC. MPL generated the schematic diagrams. Unbiased proteomics and the statistical analysis of the proteomic results were all performed by the Biological Mass Spectrometry Core Facility, at the Cancer Research UK Manchester Institute, under the supervision of DLS. The manuscript was then submitted online for independent peer review. All suggested modifications were incorporated into the final manuscript.

\section{REFERENCES}

1. Samper E, Flores JM and Blasco MA. Restoration of telomerase activity rescues chromosomal instability and premature aging in Terc-/- mice with short telomeres. EMBO reports. 2001; 2:800-807.

2. Artandi SE and DePinho RA. Telomeres and telomerase in cancer. Carcinogenesis. 2010; 31:9-18.

3. Weinrich SL, Pruzan R, Ma L, Ouellette M, Tesmer VM, Holt SE, Bodnar AG, Lichtsteiner S, Kim NW, Trager JB, Taylor RD, Carlos R, Andrews WH, Wright WE, Shay JW, Harley CB, et al. Reconstitution of human telomerase with the template RNA component hTR and the catalytic protein subunit hTRT. Nature genetics. 1997; 17:498-502.

4. Flores I, Benetti R and Blasco MA. Telomerase regulation and stem cell behaviour. Current opinion in cell biology. 2006; 18:254-260.

5. Tsai CC, Chen CL, Liu HC, Lee YT, Wang HW, Hou LT and Hung SC. Overexpression of hTERT increases stemlike properties and decreases spontaneous differentiation in human mesenchymal stem cell lines. Journal of biomedical science. 2010; 17:64

6. Zhang X, Mar V, Zhou W, Harrington L and Robinson MO. Telomere shortening and apoptosis in telomerase-inhibited human tumor cells. Genes \& development. 1999; 13:23882399.

7. Yu L, Liu S, Zhang C, Zhang B, Simoes BM, Eyre R, Liang Y, Yan H, Wu Z, Guo W and Clarke RB. Enrichment of human osteosarcoma stem cells based on hTERT transcriptional activity. Oncotarget. 2013; 4:2326-2338.

8. Mani SA, Guo W, Liao MJ, Eaton EN, Ayyanan A, Zhou AY, Brooks M, Reinhard F, Zhang CC, Shipitsin M, Campbell LL, Polyak K, Brisken C, Yang J and Weinberg
RA. The epithelial-mesenchymal transition generates cells with properties of stem cells. Cell. 2008; 133:704-715.

9. Lamb R, Harrison H, Smith DL, Townsend PA, Jackson T, Ozsvari B, Martinez-Outschoorn UE, Pestell RG, Howell A, Lisanti MP and Sotgia F. Targeting tumor-initiating cells: eliminating anabolic cancer stem cells with inhibitors of protein synthesis or by mimicking caloric restriction. Oncotarget. 2015; 6:4585-4601.

10. Casey T, Bond J, Tighe S, Hunter T, Lintault L, Patel O, Eneman J, Crocker A, White J, Tessitore J, Stanley M, Harlow S, Weaver D, Muss H and Plaut K. Molecular signatures suggest a major role for stromal cells in development of invasive breast cancer. Breast cancer research and treatment. 2009; 114:47-62.

11. Machado HL, Kittrell FS, Edwards D, White AN, Atkinson RL, Rosen JM, Medina D and Lewis MT. Separation by cell size enriches for mammary stem cell repopulation activity. Stem cells translational medicine. 2013; 2:199-203.

12. Edinger AL and Thompson CB. Akt maintains cell size and survival by increasing mTOR-dependent nutrient uptake. Molecular biology of the cell. 2002; 13:2276-2288.

13. Faridi J, Fawcett J, Wang L and Roth RA. Akt promotes increased mammalian cell size by stimulating protein synthesis and inhibiting protein degradation. American journal of physiology Endocrinology and metabolism. 2003; 285:E964-972.

14. Fingar DC, Salama S, Tsou C, Harlow E and Blenis J. Mammalian cell size is controlled by mTOR and its downstream targets S6K1 and 4EBP1/eIF4E. Genes \& development. 2002; 16:1472-1487.

15. Sahin E and Depinho RA. Linking functional decline of telomeres, mitochondria and stem cells during ageing. Nature. 2010; 464:520-528.

16. Sahin E, Colla S, Liesa M, Moslehi J, Muller FL, Guo M, Cooper M, Kotton D, Fabian AJ, Walkey C, Maser RS, Tonon G, Foerster F, Xiong R, Wang YA, Shukla SA, et al. Telomere dysfunction induces metabolic and mitochondrial compromise. Nature. 2011; 470:359-365.

17. Moslehi J, DePinho RA and Sahin E. Telomeres and mitochondria in the aging heart. Circulation research. 2012; 110:1226-1237.

18. Sahin E and DePinho RA. Axis of ageing: telomeres, p53 and mitochondria. Nature reviews Molecular cell biology. 2012; 13:397-404.

19. Hu J, Hwang SS, Liesa M, Gan B, Sahin E, Jaskelioff M, Ding Z, Ying H, Boutin AT, Zhang H, Johnson S, Ivanova E, Kost-Alimova M, Protopopov A, Wang YA, Shirihai OS, et al. Antitelomerase therapy provokes ALT and mitochondrial adaptive mechanisms in cancer. Cell. 2012; 148:651-663.

20. Ertel A, Tsirigos A, Whitaker-Menezes D, Birbe RC, Pavlides S, Martinez-Outschoorn UE, Pestell RG, Howell A, Sotgia F and Lisanti MP. Is cancer a metabolic rebellion against host aging? In the quest for immortality, tumor 
cells try to save themselves by boosting mitochondrial metabolism. Cell cycle. 11:253-263.

21. Viale A, Pettazzoni P, Lyssiotis CA, Ying H, Sanchez N, Marchesini M, Carugo A, Green T, Seth S, Giuliani V, Kost-Alimova M, Muller F, Colla S, Nezi L, Genovese G, Deem AK, et al. Oncogene ablation-resistant pancreatic cancer cells depend on mitochondrial function. Nature. 2014; 514:628-632.

22. Kawauchi K, Ihjima K and Yamada O. IL-2 increases human telomerase reverse transcriptase activity transcriptionally and posttranslationally through phosphatidylinositol 3'-kinase/Akt, heat shock protein 90, and mammalian target of rapamycin in transformed NK cells. Journal of immunology. 2005; 174:5261-5269.

23. Sundin T, Peffley DM and Hentosh P. Disruption of an hTERT-mTOR-RAPTOR protein complex by a phytochemical perillyl alcohol and rapamycin. Molecular and cellular biochemistry. 2013; 375:97-104.

24. Hosokawa M, Kenmotsu H, Koh Y, Yoshino T, Yoshikawa T, Naito T, Takahashi T, Murakami H, Nakamura Y, Tsuya A, Shukuya T, Ono A, Akamatsu H, Watanabe R, Ono S, Mori K, et al. Size-based isolation of circulating tumor cells in lung cancer patients using a microcavity array system. PloS one. 2013; 8:e67466.

25. Chen CL, Mahalingam D, Osmulski P, Jadhav RR, Wang CM, Leach RJ, Chang TC, Weitman SD, Kumar AP, Sun L, Gaczynska ME, Thompson IM and Huang TH. Singlecell analysis of circulating tumor cells identifies cumulative expression patterns of EMT-related genes in metastatic prostate cancer. The Prostate. 2013; 73:813-826.

26. Desitter I, Guerrouahen BS, Benali-Furet N, Wechsler J, Janne PA, Kuang Y, Yanagita M, Wang L, Berkowitz JA, Distel RJ and Cayre YE. A new device for rapid isolation by size and characterization of rare circulating tumor cells. Anticancer research. 2011; 31:427-441.

27. Baccelli I, Schneeweiss A, Riethdorf S, Stenzinger A, Schillert A, Vogel V, Klein C, Saini M, Bauerle T, Wallwiener M, Holland-Letz T, Hofner T, Sprick M, Scharpff M, Marme F, Sinn HP, et al. Identification of a population of blood circulating tumor cells from breast cancer patients that initiates metastasis in a xenograft assay. Nature biotechnology. 2013; 31:539-544.

28. Tan AS, Baty JW, Dong LF, Bezawork-Geleta A, Endaya B, Goodwin J, Bajzikova M, Kovarova J, Peterka M, Yan B, Pesdar EA, Sobol M, Filimonenko A, Stuart S, Vondrusova M, Kluckova K, et al. Mitochondrial genome acquisition restores respiratory function and tumorigenic potential of cancer cells without mitochondrial DNA. Cell metabolism. 2015; 21:81-94.

29. Lamb R, Harrison H, Hulit J, Smith DL, Lisanti MP and Sotgia F. Mitochondria as new therapeutic targets for eradicating cancer stem cells: Quantitative proteomics and functional validation via MCT1/2 inhibition. Oncotarget. 2014; 5:11029-11037.

30. De Luca A, Fiorillo M, Peiris-Pages M, Ozsvari B,
Smith DL, Sanchez-Alvarez R, Martinez-Outschoorn UE, Cappello AR, Pezzi V, Lisanti MP and Sotgia F. Mitochondrial biogenesis is required for the anchorageindependent survival and propagation of stem-like cancer cells. Oncotarget. 2015.

31. Lamb R, Ozsvari B, Lisanti CL, Tanowitz HB, Howell A, Martinez-Outschoorn UE, Sotgia F and Lisanti MP. Antibiotics that target mitochondria effectively eradicate cancer stem cells, across multiple tumor types: Treating cancer like an infectious disease. Oncotarget. 2015.

32. Lamb R, Fiorillo M, Chadwick A, Ozsvari B, Reeves KJ, Smith DL, Clarke RB, Howell SJ, Cappello AR, MartinezOutschoorn UE, Peiris-Pages M, Sotgia F and Lisanti MP. Doxycycline down-regulates DNA-PK and radiosensitizes tumor initiating cells: Implications for more effective radiation therapy. Oncotarget. 2015; 6:14005-14025.

33. Baselga J, Campone M, Piccart M, Burris HA, 3rd, Rugo HS, Sahmoud T, Noguchi S, Gnant M, Pritchard KI, Lebrun F, Beck JT, Ito Y, Yardley D, Deleu I, Perez A, Bachelot $\mathrm{T}$, et al. Everolimus in postmenopausal hormone-receptorpositive advanced breast cancer. The New England journal of medicine. 2012; 366:520-529.

34. Shaw FL, Harrison H, Spence K, Ablett MP, Simoes BM, Farnie $\mathrm{G}$ and Clarke RB. A detailed mammosphere assay protocol for the quantification of breast stem cell activity. Journal of mammary gland biology and neoplasia. 2012; 17:111-117.

35. Holland M, Castro FV, Alexander S, Smith D, Liu J, Walker M, Bitton D, Mulryan K, Ashton G, Blaylock M, Bagley S, Connolly Y, Bridgeman J, Miller C, Krishnan S, Dempsey $\mathrm{C}$, et al. RAC2, AEP, and ICAM1 expression are associated with CNS disease in a mouse model of pre-B childhood acute lymphoblastic leukemia. Blood. 2011; 118:638-649. 